\title{
ANALYSIS OF THE SOFT X-RAY PULSATIONS OF DWARF NOVAE
}

\author{
F. A. Cordova
}

Physics Department

California Institute of Technology

Both SS Cyg and U Gem show enhanced soft X-ray emission (0.1-0.5 $\mathrm{keV}$ ) during optical outbursts. In extended HEAO-1 pointings, it was discovered that a large fraction of this emission is pulsed (see Fig. 1). This confirms the general expectation that high energy pulsations are the ultimate source of the optical pulsations observed during outbursts of dwarf novae. For SS Cyg the average soft $X$-ray pulsation amplitude 1s $30 \%$; this is to be contrasted with the upper 1imit of $10 \%$ to a hard X-ray (2-25 keV) pulsed component for the same observation (Swank 1979). Thus the origin of the oscillation is in soft X-rays, and the direct Implication of such a large pulsed amplitude is that the oscillation itself must be an intrinsic part of the X-ray production mechanism.

Power spectra of relatively short stretches of the data for both systems are shown in Figs. 2 and 3 . Most of the power in SS Cyg's slgnal lies in a narrow frequency interval, while for $U \mathrm{Gem}$ the power

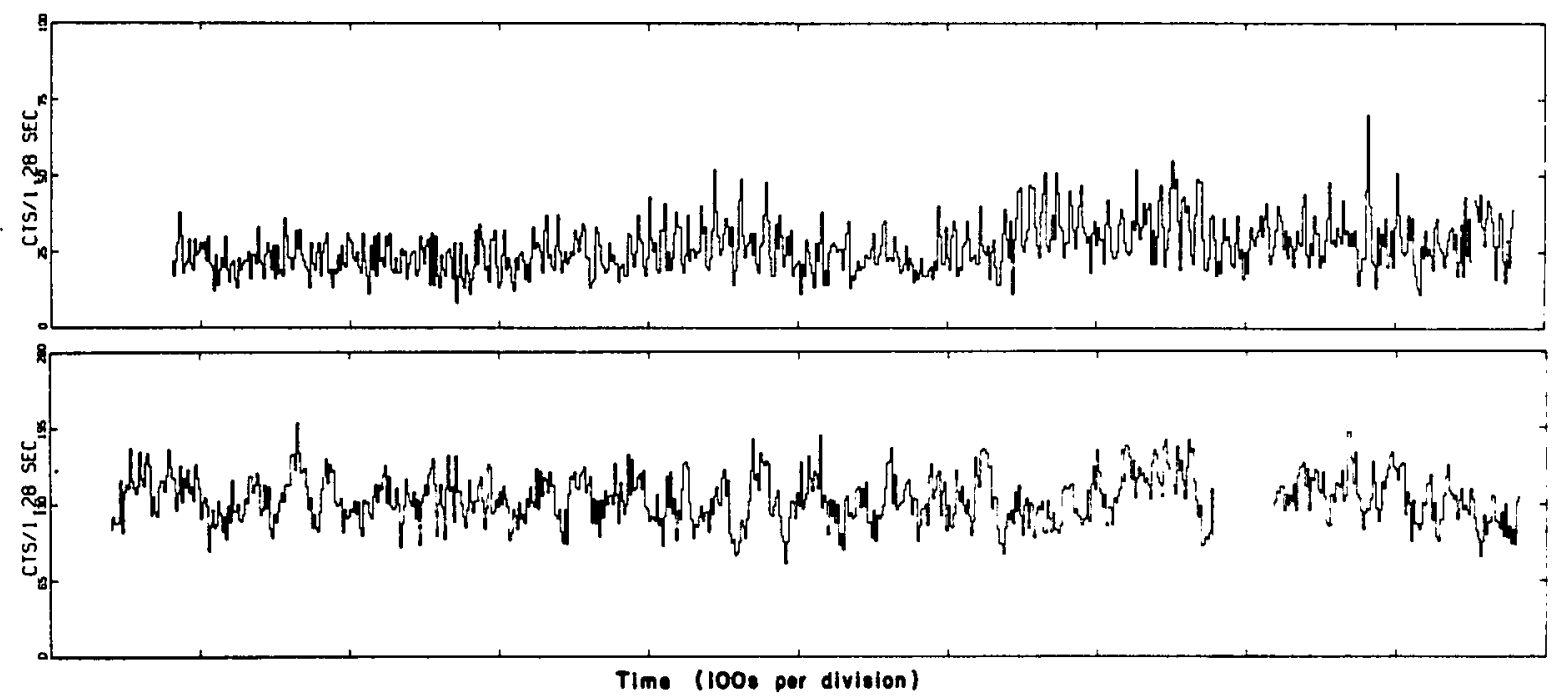

Fig. 1 - A portion of the soft X-ray light curves of both dwarf novae. Aside from amplitude modulation, the pulsations of SS Cyg look falrly regular. U Gem's pulsations look chaotic by comparison, having no well-defined period. 

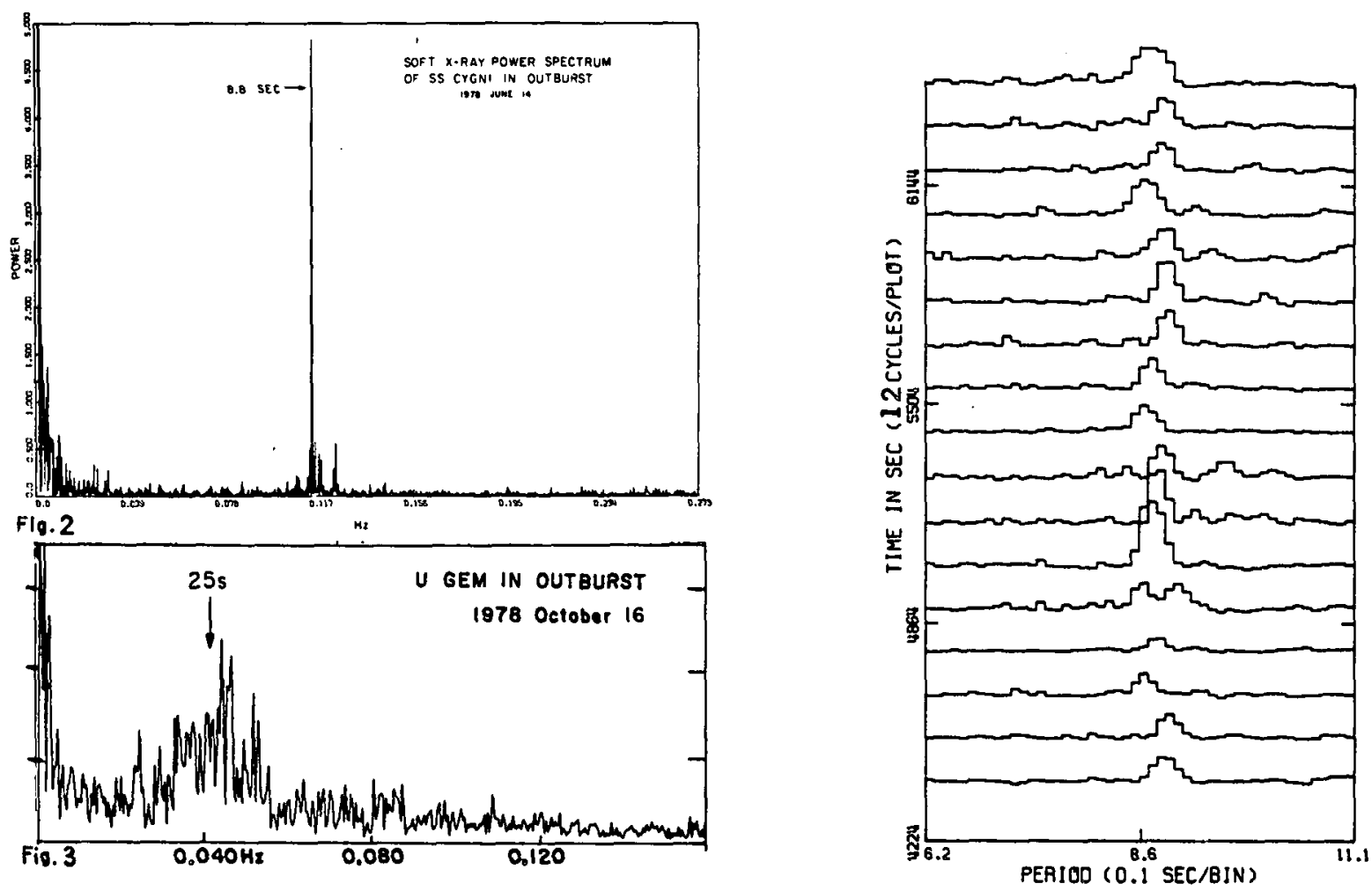

FIg. 4 - SS Cyg perlodogram

is broadly distributed around a mean period of $25 \mathrm{~s}$. It is difficult to look for changes on short timescales using the Fourler transform technique. If frequency or phase nolse is present, it is also not possible to meaningfully search for harmonics.

Conventional analysis of the optical oscillations from cataclysmic variables make use of the "perlodogram" technique; that is, short segments of continuous data are folded on a mean period and the amplitudes are plotted versus period. Such a technique applled to SS Cyg's soft $X$-ray pulsations; and illustrated for a portion of the data in Fig. 4, show that the $X-r a y$ pulsations have the same characterlstics as the optical pulsations observed in many dwarf novae: large amplitude variability, period excursions on short timescales, and the occasional presence of multiple periods. When this technique is applied to all the SS Cyg X-ray data, a long term perlod drift amounting to $-1 \times 10^{-5}$ $s s^{-1}$ is evident. The problem with this type of analysis is that an apparent change in period, such as the small shifts observed from one perlodogram to the next in Fig. 4, can be produced by variations in either phase or amplitude, or both; it is not possible to distinguish the true nature of the noise component.

The technique we eventually settled on to analyze the pulsations was the following.* We determined the pulse arrival times by crosscorrelating individual pulses or groups of pulses with a master pulse profile (for SS Cyg a sinusold was used because it best approximated the pulse shape observed in foldings of the data). We thus obtained

\footnotetext{
* A detalled analysis of the pulsations is described in C6rdova et al. $(1979 a, b)$.
} 
both the amplitude and phase of the pulsation as a function of time. We found that the amplitude can change by a factor of six within fifteen pulses with no effect on the pulse phase. The phase itself exhibits both slow varlations and rapld jumps. In Fig. 5 these results are illustrated for a portion of the SS Cyg data.

By superposing each pulse so that the maxima colnclde, we were able to sensitively search for harmonics. The pulsation

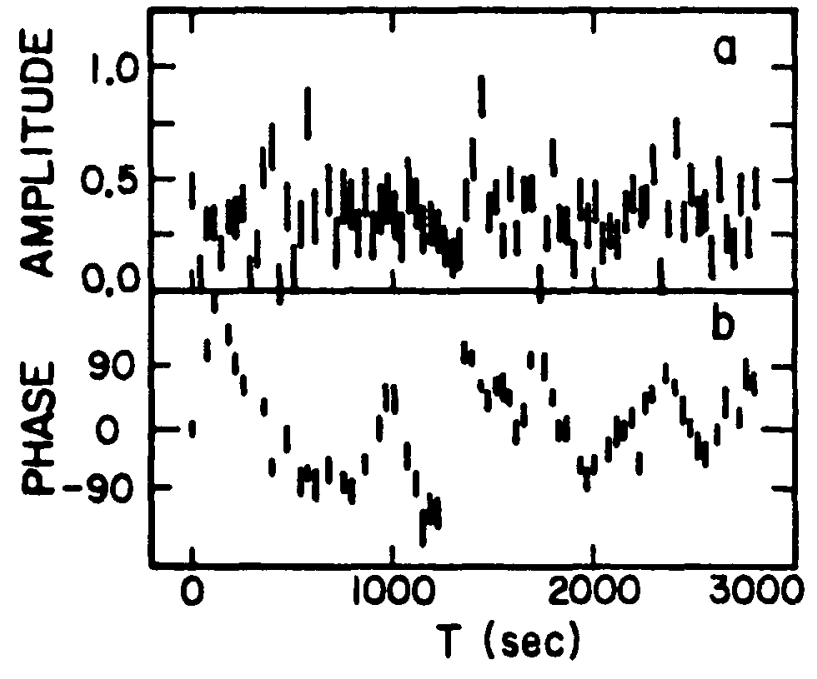

F1g. 5 Is remarkably sinusoldal, with an upper 1 inlt of $12 \%$ for the first harmonic and $\sim 7 \%$ for other harmonics.

Our chief intention was to investigate the noise properties of the oscillation, that is, to determine whether the pulsed signal was dominated by "phase noise," "frequency noise," or "slowing-down noise" (see Groth 1971). We did this by examining how the variance of the phase about a constant period depends on time. Figure 6 shows the result, namely that the varlance increases approximately inearly with time. This is consistent with a random walk in phase caused by white noise in the period of the oscillation.

The slope of the phase varlance versus time would give us the strength of the random walk if we knew the underlying pulsation period. Since we do not have this knowledge we, instead, simulate the data by generating arrival times $T_{N}$ such that $T_{N}=T_{N}+P$, where $P=P_{V_{1}}+P_{1} x$, $P_{0}$ and $P_{1}$ are fixed numbers, and $x$ is a normalfy distributed random variable with a mean of zero and unit standard deviation.

For $P_{0}=8.77 \mathrm{~g}$ (chosen somewhat arbitrarily), we find that $P_{1}=0.04 \pm 0.06 \mathrm{~s}$ produces a curve consistent with Fig. 6.

Although we know that phase steps must occur at least as often as once per cycle, we do not know the true frequency of the steps. Thus, Instead of spectific values of $P_{0}$ and $P_{1}$, our simulation gives us the strength of the random walk, which is defined as a rate of phase steps times the mean square phase step:

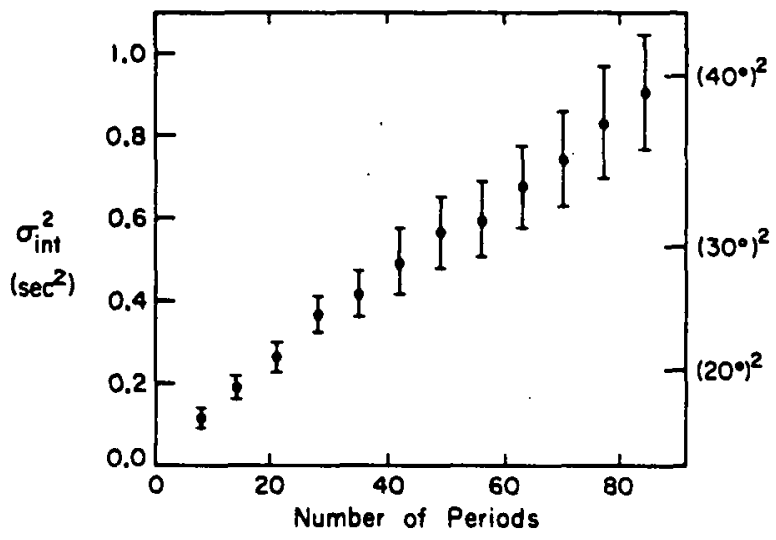

F1g. 6 


$$
S=R\left\langle(\Delta \phi)^{2}\right\rangle=\frac{1}{P_{0}}\left(\frac{2 \pi P_{1}}{P_{0}}\right)^{2}=0.011 \pm 0.003 s^{-1}
$$

In a random walk of this strength, the phase of the oscillation changes by $90^{\circ}$ on the average every $25 \pm 7$ pulses!

In a similar analysis performed on U Gem's oscillations we find that the intrinsic varlance of the phase also grows linearly with time, and that $P_{0}=25 \mathrm{~s}, P_{1}=5 \pm 1$ s reproduces the varlance in slope. Although superficially the quasi-coherent pulsation from U Gem seems qualitatively different from the more coherent soft X-ray pulsation of SS Cygni, it appears that a change in only the strength of the random walk in phase can account for the difference between the two stars; our analysis shows that the phase noise in U Gem is $\sim 10-30$ times greater than in SS Cyg.

To demonstrate in a simple way the characteristics of a pulsed signal subject to phase noise, we have generated artificlal data sets showing what happens to a pulsation when the strength of the random walk is Increased. Figure 7 shows three power spectra generated by

$$
f(t)=100+20 \cos \left(\omega t+\phi_{t}\right)+20 \cos \left(2 \omega\left(t+\phi_{t}\right)\right),
$$

where $\phi_{t}$ is a step function which changes its value every cycle:

$$
\phi_{t}=\phi_{t-P_{0}}+\frac{2 \pi P_{1}}{P_{0}} x \text {. }
$$

Period (5)

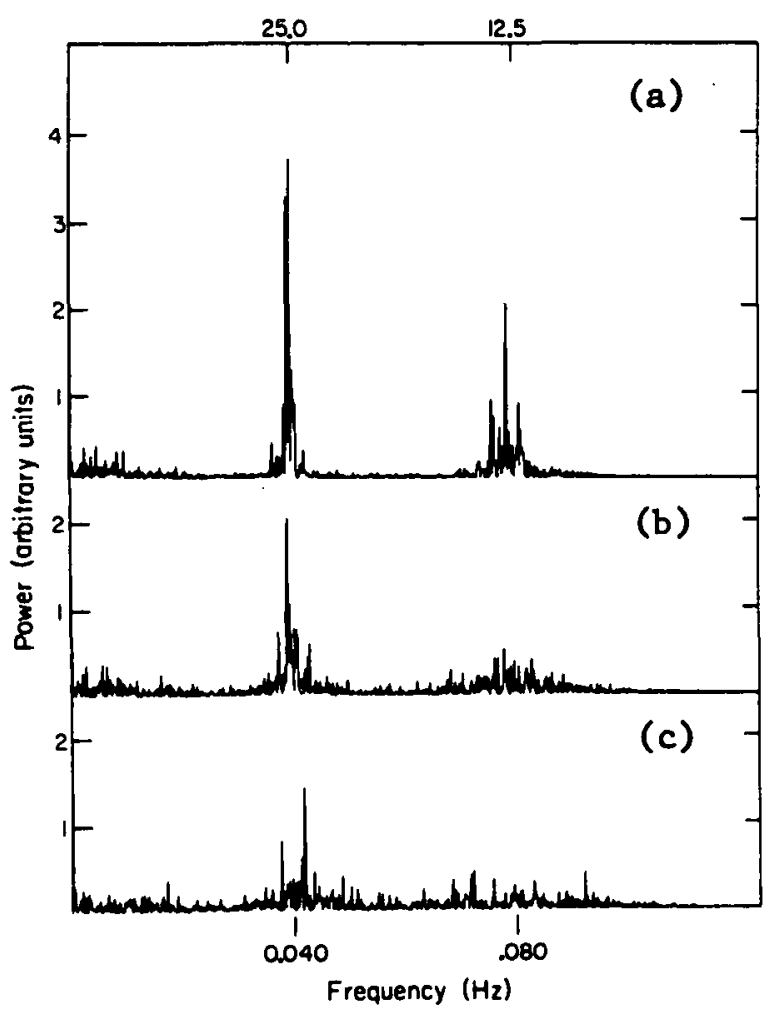

$P_{0}$ is the basic pulsation period, chosen to be $25 \mathrm{~s}$.

We have chosen values for $P_{1}$, the standard deviation of $P_{0}$, equal to $1.5,3$, and $5 \mathrm{in} \mathrm{F1g.}$ $7 a, b$, and $c$, respectively. Our artificial data set consisted of 256 pulses. The figure 11lustrates several important points :

1. A variation in the strength of the random walk can change the "coherent" pulsation of SS Cyg to the "Incoherent" pulsation of U Gem. Fig. 7a shows a strong pulsation at $25 \mathrm{~s}$ with an appearance simflar to that for SS Cyg. Fig. 7c, on 
the other hand, shows only an enhancement in the power spectrum at periods clustering around $25 \mathrm{~s}$, a profile similar to that for U Gem.

2. Harmonics will be even more strongly affected by phase noise than the fundamental is affected. In (c) the harmonic that showed up strongly in (a) has completely disappeared even though it has an amplitude equal to that of the fundamental! Th1s could explain the absence of harmonics in the observed dwarf novae pulsations.

3. The phase nolse produces random period shifts. In (a), where the phase noise was weak, the peak is $0.1 \mathrm{~s}$ away from the true pulse period. In (b) it is shifted by $0.2 \mathrm{~s}$.

4. The random walk can produce multiple pseudo-periodicities. (see the two spikes in Fig. 7c).

Thus the behavior of the so-called "coherent" visual osctllations (Patterson, Robinson, and Nather 1977; Patterson, Robinson, and Kiplinger 1978) can be explained by intrinsic phase instability; these pulsations resemble the X-ray pulsations in SS Cyg. The "quasi-coherent" visual oscillations observed for several dwarf novae on the decline from maximum brightness (Robinson and Nather 1979) have a behavior similar to the X-ray pulsation in U Gem. Figure 7 shows that the difference between the "coherent" and "quasi-coherent" pulsations may be only in the strength of the random walk in phase. It is possible that all of the optical and X-ray oscillations are produced by the same mechanism.

\section{ACKNOWLEDGEMENTS}

Dr. Thomas Chester provided invaluable contributions to the timing analysis. Dr. Gordon Garmire provided helpful critique throughout the Investigation. This work was supported by NASA contract NAS 5-25049.

\section{REFERENCES}

Cordova, F. A., Chester, T. J., Tuohy, I. R., and Garmire, G. P. 1979a, Ap. J., in press.

Cordova, F. A., Chester, T. J., and Garmire, G. P. 1979b, in preparation.

Groth, E. J. 1971, Ph.D. Thesis, Princeton University.

Patterson, J., Robinson, E. L., and Kiplinger, A. L. 1978, Ap. J. (Letters), 226, L137.

Patterson, J., Robinson, E. I., and Nather, R. E. 1977, Ap. J., 214, 144.

Robinson, E. L., and Nather, R. E. 1979, Ap. J. Supp1., 39, No. 4.

Swank, J. H. 1979, private communication. 